\title{
Immunocytochemical mapping of the phosphatase and tensin homolog (PTEN/MMAC1) tumor suppressor protein in human gliomas ${ }^{1}$
}

\author{
Dan Fults ${ }^{2}$ and Carolyn Pedone \\ Department of Neurosurgery and Huntsman Cancer Institute, University of Utah Health Sciences Center, \\ Salt Lake City, UT 84132
}

PTEN/MMAC1 (phosphatase and tensin homolog/ mutated in multiple advanced cancers 1 ) is a tumor suppressor gene, the inactivation of which is an important step in the progression of gliomas to end-stage glioblastoma multiforme. We examined the distribution of PTEN protein in 49 primary human gliomas by immunocytochemistry using polyclonal antibodies that we raised against PTEN-glutathione S-transferase fusion proteins expressed in Escherichia coli. The study group consisted of 6 low-grade astrocytomas, 7 anaplastic astrocytomas, 21 glioblastomas multiforme, 4 low-grade oligodendrogliomas, 6 malignant oligodendrogliomas, and 5 malignant mixed oligoastrocytomas. For each tumor, we determined the percentage of tumor cells showing PTEN immunoreactivity in the most cellular regions of the tumor specimen. In both astrocytomas and oligodendrogliomas, there was an inverse relationship between the percentage of $\mathrm{PTEN}^{+}$cells and malignancy grade, consistent with a role for PTEN as a tumor suppressor gene, the expression of which declines during glioma progression. In nonneoplastic tissue, PTEN was expressed in human fetal brain at 16, 23, and 27 weeks' gestation, but not in adult brain, indicating that PTEN is developmentally regulated in the CNS. In 21 glioblastomas multiforme, we

\footnotetext{
Received 25 June 1999, accepted 29 October 1999.
}

${ }^{1}$ This work was supported by grants from the National Institutes of Health (CA52855) and the Huntsman Cancer Foundation.

${ }^{2}$ Address correspondence and reprint requests to Dan Fults, Department of Neurosurgery, University of Utah Health Sciences Center, Salt Lake City, UT 84132.

${ }^{3}$ Abbreviations used are as follows: GBM, glioblastoma multiforma; GFP, green fluorescent protein; GST, glutathione S-transferase; LOH, loss of heterozygosity; PTEN, phosphatase and tensin homolog. correlated PTEN protein expression with PTEN gene sequence. Although PTEN-mutant tumors showed significantly diminished PTEN protein expression compared with wild-type cases, suppressed expression of PTEN is more prevalent than predicted from mutation frequencies. Neuro-Oncology 2, 71-79, 2000 (Posted to NeuroOncology [serial online], Doc. 99-28, March 22, 2000. URL <neuro-oncology.mc.duke.edu>)

$A$ strocytomas, the most frequently occurring gliomas, and the less common oligodendrogliomas are thought to arise by transformation of astrocytes and oligodendrocytes, respectively. The fact that many gliomas are mixtures of astrocytic and oligodendroglial cells suggests that some gliomas may arise not from differentiated glia but from multipotential glial stem cells (Noble, 1996). Regardless of their cell of origin, gliomas all tend to progress through stages of increasing malignancy with the passage of time. Glioma progression has been studied most extensively in tumors of astrocytic lineage where at least four stages can be identified histologically: two benign stages, grade I (pilocytic) and grade II astrocytoma; an intermediate malignant stage, grade III anaplastic astrocytoma; and an end stage, grade IV GBM. ${ }^{3}$

Tumor progression is a multistep process driven by accumulation of somatic mutations in various genes controlling cell growth. Several groups of investigators have identified a tumor suppressor gene, PTEN (also called MMAC1 [mutated in multiple advanced cancers 1], or TEP1 [telomerase-associated protein 1]), located on chromosome 10, the inactivation of which is an important step in GBM formation (Li and Sun, 1997; Li et al., 1997; Steck et al., 1997). PTEN has dual-specificity phosphatase activity in vitro against peptide substrates (Myers et al., 1997). A growing body of experimental evidence 
indicates that PTEN inhibits cell growth via the protein kinase $\mathrm{B}(\mathrm{PKB}, \mathrm{Akt})$ signal transduction pathway. PTEN dephosphorylates the lipid second messenger, phosphatidylinositol 3,4,5-trisphosphate (PIP3) (Maehama and Dixon, 1998). Mutations that eliminate the lipid phosphatase activity of PTEN also reduce its ability to suppress cell growth (Furnari et al., 1998). Glioblastoma cells with PTEN mutations and thymic lymphomas that arise in $\mathrm{Pten}^{+-}$transgenic mice show increased activity of protein kinase B and elevated levels of phosphatidylinositol 3,4,5-trisphosphate (Haas-Kogan et al., 1998; Suzuki et al., 1998).

The N-terminal half of PTEN shares close sequence homology with the cytoskeletal proteins tensin and auxilin, suggesting a role in adhesion-dependent cell signaling. Transfection experiments have shown that overexpression of PTEN reduces cell migration, cell spreading, focal adhesion formation, and actin stress fiber formation (Tamura et al., 1998).

Sequence analysis of the PTEN coding region has shown that mutations are present in $24 \%$ to $44 \%$ of primary GBMs (Fults et al., 1998; Liu et al., 1997; Rasheed et al., 1997; Wang et al., 1997). The fact that the frequency of $\mathrm{LOH}$ on chromosome 10 reported in GBM patients $(>70 \%)$ is much higher than the above-described frequency of PTEN gene mutation suggests that other tumor suppressor genes exist on chromosome 10 or that PTEN expression is lost in tumors without a coding sequence mutation. A study using reverse transcription-polymerase chain reaction and immunocytochemistry showed PTEN expression in GBMs to be diminished or absent in $69 \%$ of cases - higher than mutation frequencies reported previously (Sano et al., 1999). In that study, PTEN expression was detected in vascular endothelial cells and neurons. That observation implies that the overall level of mRNA or protein in a tumor sample may not accurately reflect gene expression in the tumor cell population.

To examine the distribution of PTEN protein in tumor cells, we carried out an immunocytochemistry study of 49 primary human gliomas, including 21 GBMs with a known PTEN gene sequence. In both astrocytomas and oligodendrogliomas, PTEN expression was lower in high-grade tumors, consistent with its role as a tumor suppressor. PTEN was expressed in human fetal brain but not in adult brain, indicating that PTEN is developmentally regulated in the CNS. Correlation of PTEN genotype and expression in GBMs showed that suppressed expression of PTEN was common, even in cases without a PTEN gene mutation.

\section{Materials and Methods}

\section{Preparation of Antisera}

We expressed two regions of wild-type, human PTEN cDNA in E. coli as GST fusion proteins using the bacterial expression vector pGEX-2T (Pharmacia Biotech, Piscataway, N.J.). These regions corresponded to N-terminal amino acids M35-L100 and C-terminal amino acids Y336-V403. Polyclonal antisera were raised in rabbits against affinity-purified fusion proteins (Cov- ance Research Products, Inc., Denver, PA). Two rabbits were immunized with each antigen. We tested immune antisera obtained from each rabbit 72 days postvaccination (after the primary immunization and three boosts) by immunoblotting against purified fusion proteins. Antibodies directed against GST were blocked by preadsorbing immune sera with sonicated extracts of bacteria carrying the nonrecombinant pGEX-2T vector (no PTEN cDNA insert present) and expressing only GST. Each immune serum recognized a protein of the same molecular weight as the original antigen. To verify that an immune response was occurring specifically against the PTEN sequence, we digested the fusion proteins with thrombin protease to cleave off the GST peptide segment and analyzed the cleavage products on Western blots with the immune antisera. In each case, the PTEN cleavage fragments were recognized by immune antisera, and no immunoreactivity was seen when we probed with preimmune sera.

To demonstrate the specificity of the antisera against PTEN, we coupled $100 \mu \mathrm{g}(2.8 \mathrm{nmol})$ of affinity-purified antigen (39-kDa PTEN-GST fusion protein containing the C-terminal portion of PTEN fused to GST) to glutathione Sepharose-4B beads for $20 \mathrm{~min}$. The beads were washed three times with phosphate-buffered saline, then collected by centrifugation. Antiserum was diluted 1:100 in phosphate-buffered saline, and $100 \mu \mathrm{l}$ of diluted antiserum (10 $\mu \mathrm{g}$ of $\mathrm{IgG}, 0.063 \mathrm{nmol})$ was incubated with the Sepharose-coupled PTEN-GST antigen for $60 \mathrm{~min}$ on ice. Antigen-antibody complexes, coupled to the Sepharose beads, were removed by centrifugation, and the supernatant was diluted 1:40 with phosphate-buffered saline. This adsorbed antiserum was then applied to Western transfer filters to demonstrate complete blocking of immunoreactivity against the 77-kDa PTEN-GFP fusion protein expressed by COS-7 cells (Fig. 1C). The final antiserum dilution was 1:4000, identical to that used for immunostaining the tissue sections. In control adsorptions, we repeated the above-described protocol using $100 \mu \mathrm{g}(3.8 \mathrm{nmol})$ of affinity-purified GST or $20 \mu \mathrm{l}$ of phosphate-buffered saline instead of PTEN-GST.

\section{Transient Transfection of COS-7 Cells}

For each transfection, COS-7 cells $\left(2 \times 10^{6}\right)$ were plated in medium containing $10 \%$ fetal bovine serum and incubated at $37^{\circ} \mathrm{C}$ until cell density reached $70 \%$ confluence. Expression plasmid DNA (16 $\mu \mathrm{g}$ in $800 \mu$ l OptiMEM medium) was mixed with $15 \mu$ l LipofectAMINE (GibcoBRL, Grand Island, N.Y.) and incubated at room temperature for 30 min. Recipient cells were washed in serum-free medium, and the LipofectAMINE/DNA mixture was added. After the cells were incubated at $37^{\circ} \mathrm{C}$ for $5 \mathrm{~h}$ in the absence of serum, an equal volume of growth medium containing $20 \%$ fetal bovine serum was added, and the transfected cells were incubated for an additional $48 \mathrm{~h}$. In each experiment, we assessed transfection efficiency by transfecting a control plate of cells with pEGFP-C1 (Clontech, Palo Alto, CA), a reporter vector expressing GFP. By counting the number of GFP cells, we estimated an average transfection efficiency of $30 \%$. The PTEN expression plasmids we 


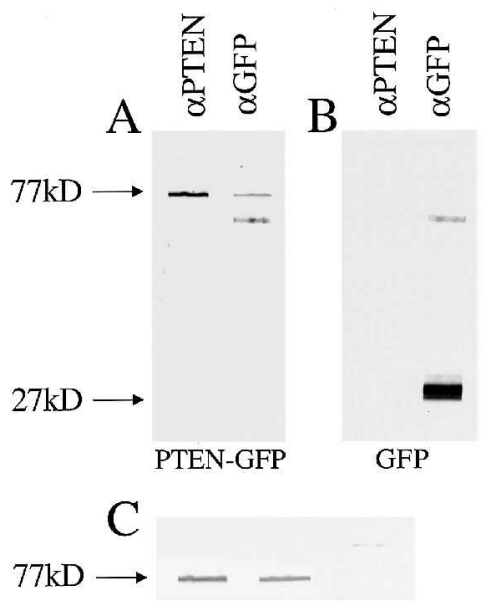

\section{GST PTEN-GST}

Fig. 1. Western analysis of COS-7 cells. A and B. COS-7 cells overexpressing PTEN-GFP fusion protein (A) or GFP monomer (B). AntiPTEN antisera (1:4000) detected a 77-kDa fusion protein in cells expressing PTEN-GFP, but not in cells expressing GFP alone. AntiGFP antisera (1:1000) detected the 77-kDa PTEN-GFP fusion protein and the $27-k D a$ GFP moiety. C. Immunoreactivity against the $77-k D a$ fusion protein was completely blocked by preadsorption with the original immunizing antigen (PTEN-GST), but not with GST alone or phosphate buffered saline (0).

tested were as follows: pEGFP-PTEN containing fulllength, wild-type, human PTEN cDNA fused $\mathbf{5}^{\prime}$ to GFP in expression vector pEGFP-C1; and pEFm-PTEN containing the same PTEN cDNA with 5' CMYC epitope tag in expression vector $\mathrm{pEFm}$ (gifts from Dr. Andrew Thorburn, University of Utah).

\section{Patient Study Group}

Tumor samples were obtained from the surgical pathology archives of the University of Utah Health Sciences Center. The tumor tissue had been fixed in formalin at the time of surgery, then embedded in paraffin. Tumors were classified according to the World Health Organization international histologic classification (Kleihues et al., 1993). Patient selection was restricted to cases where an extensive tumor resection had been performed so as to minimize the sampling error inherent in needle biopsy procedures. The age and sex of the 49 patients are shown in Table 1. All tumors were removed at surgery, prior to radiation or chemotherapy, except in 9 patients (A36, A34, U48, U71, U58, O18, O76, O22, A26) who had received prior radiation and/or chemotherapy.

When subdivided according to tumor histopathology, the patient study group had 6 low-grade astrocytomas (pilocytic astrocytomas and grade 2 fibrillary astrocytomas), 7 anaplastic astrocytomas, 21 GBMs, 4 low-grade oligodendrogliomas, 6 malignant oligodendrogliomas, and 5 malignant mixed oligoastrocytomas. Among these subgroups, there was no evidence that the groups differed with respect to sex or history of prior radiation/chemotherapy $(P=0.38$ and $P=0.45$, respectively, using the Fisher-Freeman exact test). The small size of the patient subgroups may have masked actual differences in sex or in treatment history. However, the age distribution was significantly different, with higher age associated with more advanced tumors $(P=0.0003$, using Kruskall-Wallis test). In every GBM patient, the sequence of all nine coding exons of the PTEN gene was determined, and $\mathrm{LOH}$ analysis was performed for multiple loci on chromosome 10 as reported by us previously (Fults and Pedone, 1993; Fults et al., 1998).

\section{Immunocytochemistry}

Tissue sections were cut $6-\mu \mathrm{m}$ thick, mounted on glass slides, deparaffinized with toluene, and then hydrated through a descending series of ethanol. For each tumor, sections were treated with and without an antigen unmasking protocol (Vector Laboratories, Burlingame, $\mathrm{CA}$ ) in which slides were exposed to two power bursts in a microwave oven (Sears Kenmore, Chicago, Ill.) at full power $(750 \mathrm{~W})$ for $5 \mathrm{~min}$ each. The antigen unmasking protocol heightened the intensity of the specific immunoperoxidase staining. In no case did this protocol reveal immunoreactivity staining that was not visible at lower intensity levels in untreated sections.

Sections were treated with $\mathrm{H}_{2} \mathrm{O}_{2}(1 \% \mathrm{vol} / \mathrm{vol})$ for 10 min to quench endogenous peroxidase activity. After immersion in normal goat serum $(2 \%)$, sections were incubated with primary antibody (antiserum, diluted $1: 4000-1: 2000$, or purified $\operatorname{IgG}, 3-6 \mu \mathrm{g} / \mathrm{ml}$ ) in a humid chamber at $4^{\circ} \mathrm{C}$ overnight. The avidin-biotin complex technique (Hsu et al., 1981) was used to localize PTEN immunoreactivity. Sections were developed with diaminobenzidine $(0.075 \% \mathrm{wt} / \mathrm{vol})-\mathrm{H}_{2} \mathrm{O}_{2}(0.002 \% \mathrm{vol} / \mathrm{vol})$, then counterstained with toluidine blue $(0.1 \% \mathrm{wt} / \mathrm{vol})$. To demonstrate competition, we preincubated $100 \mu \mathrm{l}$ antiserum $(1 \mathrm{mg}$ of $\mathrm{IgG}, 6.3 \mu \mathrm{mol})$ with $300 \mu \mathrm{g}(7.7 \mu \mathrm{mol})$ antigen $(39-\mathrm{kDa}$ PTEN-GST fusion protein containing the C-terminal portion of PTEN coupled to GST) for $1 \mathrm{~h}$ before diluting and applying it to the slides.

For each patient, the percentage of tumor cells showing PTEN immunoreactivity was counted in three, $40 \times$ microscope fields in the most cellular region of the tissue section. Some tumors rated at $0 \%$ contained scattered foci of positive cells remote from the tumor center. The PTEN immunoreactivity data shown in Table 1 was derived from tissue sections treated with the unmasking protocol because of the more obvious differences between $\mathrm{PTEN}^{+}$and $\mathrm{PTEN}^{-}$tumor cells.

\section{Results}

\section{Characterizing the Immune Antisera}

PTEN was overexpressed in COS-7 cells as a fusion protein coupled with GFP by transient transfection of cDNA using expression plasmid pEGFP-C1. On immunoblots, antisera directed against two different PTEN epitopes (peptide segments M35-L100 and Y336-V403) detected a $77-\mathrm{kDa}$ fusion protein in cells transfected with the fusion construct (pEGFP-PTEN) but not in cells transfected with control plasmid (pEGFP) containing GFP 
D. Fults and C. Pedone: PTEN/MMAC1 expression in gliomas

Table 1. PTEN expression in 49 human gliomas

\begin{tabular}{|c|c|c|c|c|c|}
\hline Patient & Tumor type & Sex/age & $\%$ PTEN + cells & LOH 10 & PTEN genotype \\
\hline L9 & PA & $F / 36$ & 100 & $\mathrm{~N}$ & \\
\hline L27 & PA & $M / 25$ & 100 & & \\
\hline L28 & PA & $M / 22$ & 100 & & \\
\hline L29 & PA & $M / 26$ & 100 & & \\
\hline A29 & All & $\mathrm{F} / 25$ & 83 & $\mathrm{~N}$ & \\
\hline L30 & All & $M / 26$ & 100 & & \\
\hline A28 & AA & $M / 28$ & 62 & $\mathrm{~N}$ & \\
\hline A27 & AA & $\mathrm{F} / 24$ & 0 & $\mathrm{~N}$ & \\
\hline A21 & AA & $F / 29$ & 63 & $\mathrm{~N}$ & \\
\hline A36 & AA & $F / 24$ & 26 & $\mathrm{~N}$ & \\
\hline A31 & AA & $M / 60$ & 33 & $\mathrm{~N}$ & \\
\hline A38 & AA & $M / 16$ & 50 & $\mathrm{~N}$ & \\
\hline A34 & $A A$ & $F / 42$ & 65 & $\mathrm{~N}$ & \\
\hline U49 & GBM & $M / 68$ & 0 & $\mathrm{~N}$ & Y16 > Stop (exon 1) \\
\hline U48 & GBM & $M / 33$ & 0 & $\mathrm{~N}$ & S229 > Stop (exon 7) \\
\hline U72 & GBM & $M / 59$ & 0 & $\mathrm{~N}$ & R335 > Stop (exon 8) \\
\hline U42 & GBM & $M / 82$ & 0 & $\mathrm{~N}$ & Frameshift (exon 2) \\
\hline U71 & GBM & $M / 40$ & 0 & $\mathrm{~N}$ & Splice variant (intron 4) \\
\hline U23 & GBM & $F / 62$ & 0 & $\mathrm{~N}$ & Splice variant (intron 6) \\
\hline U52 & GBM & $M / 55$ & 0 & $\mathrm{~N}$ & H61 > R (exon 3) \\
\hline U80 & GBM & $M / 56$ & 0 & $\mathrm{~N}$ & L152 > V (exon 5) \\
\hline U50 & GBM & $F / 58$ & 0 & $\mathrm{~N}$ & R173 > C (exon 6) \\
\hline U15 & GBM & $M / 71$ & 31 & $\mathrm{~N}$ & M35 > R (exon 2) \\
\hline U58 & GBM & $\mathrm{F} / 47$ & 0 & $\mathrm{~N}$ & Wild type \\
\hline U60 & GBM & $F / 58$ & 32 & $\mathrm{~N}$ & Wild type \\
\hline U68 & GBM & $M / 55$ & 0 & $\mathrm{~N}$ & Wild type \\
\hline U61 & GBM & $M / 40$ & 23 & $\mathrm{~N}$ & Wild type \\
\hline U57 & GBM & $M / 60$ & 0 & $\mathrm{~N}$ & Wild type \\
\hline U73 & GBM & $M / 49$ & 0 & $\mathrm{~N}$ & Wild type \\
\hline U70 & GBM & $M / 62$ & 0 & $\mathrm{~N}$ & Wild type \\
\hline U78 & GBM & $M / 48$ & 10 & $\mathrm{~N}$ & Wild type \\
\hline U69 & GBM & $M / 52$ & 10 & $N$ & Wild type \\
\hline U46 & GBM & $M / 28$ & 67 & $\mathrm{~N}$ & Wild type \\
\hline U79 & GBM & $M / 67$ & 55 & $Y$ & Wild type \\
\hline O18 & LGO & $F / 33$ & 100 & & \\
\hline O19 & LGO & $M / 34$ & 52 & & \\
\hline O20 & LGO & $F / 39$ & 37 & & \\
\hline $\mathrm{O} 21$ & LGO & $M / 56$ & 60 & & \\
\hline O56 & MO & $F / 45$ & 0 & $\mathrm{~N}$ & \\
\hline O5 & MO & $M / 34$ & 0 & $\mathrm{~N}$ & \\
\hline O11 & MO & $F / 56$ & 11 & $Y$ & \\
\hline O14 & MO & $M / 43$ & 7 & & \\
\hline 015 & MO & $M / 69$ & 16 & & \\
\hline O76 & MO & $M / 45$ & 19 & $\mathrm{~N}$ & \\
\hline $\mathrm{O} 22$ & $M O A$ & $M / 25$ & 10 & & \\
\hline $\mathrm{O} 23$ & $M O A$ & $M / 29$ & 31 & & \\
\hline O1 & $M O A$ & $M / 40$ & 22 & $\mathrm{~N}$ & \\
\hline A26 & MOA & $\mathrm{F} / 55$ & 50 & $\mathrm{~N}$ & \\
\hline A19 & MOA & $\mathrm{F} / 37$ & 53 & $\mathrm{~N}$ & \\
\hline
\end{tabular}

Abbreviations: PA, pilocytic astrocytoma; All, grade II astrocytoma; AA, anaplastic astrocytoma; GBM, glioblastoma multiforme; LGO, low grade oligodendroglioma; MO, malignant oligodendroglioma; MOA, malignant mixed oligoastrocytoma. 
alone (Fig. 1A). Control antisera against GFP detected the $77-\mathrm{kDa}$ PTEN-GFP fusion protein and the $27-\mathrm{kDa}$ GFP moiety in cells transfected with pEGFP-PTEN and pEGFP, respectively (Fig. 1B). The anti-GFP serum detected an additional band in the 65 - to $70-\mathrm{kDa}$ size range in COS-7 cells transfected with PTEN-GFP, GFP, or salmon sperm DNA. This band most likely represents cross reactivity with an unknown COS-7 cell protein unrelated to PTEN.

To control for effects on epitope presentation by the 27-kDa GFP fusion partner, we transfected COS-7 cells with full-length PTEN cDNA carrying a short CMYC epitope tag (10 amino acid residues). On immunoblots, all four anti-PTEN antisera and a control monoclonal antibody specific for the CMYC epitope detected a 50$\mathrm{kDa}$ PTEN-CMYC fusion protein (data not shown).

Immunoreactivity against the $77-\mathrm{kDa}$ PTEN-GFP fusion protein expressed by COS-7 cells was completely blocked by preadsorption of antiserum with the original immunizing antigen. Fig. 1C shows the results of an adsorption (competition) experiment using antibody UT139 directed against a 39-kDa PTEN-GST fusion protein containing the C-terminal portion of PTEN. We concluded from these results that our antisera recognized PTEN specifically.

\section{PTEN Immunocytochemistry in Human Gliomas}

We examined PTEN protein expression in a series of 49 human gliomas by immunoperoxidase staining of formalin-fixed tissue sections. For each tumor, we determined the percentage of tumor cells showing PTEN immunoreactivity in the most cellular regions of the tumor specimen. The data shown in Table 1 were obtained following antigen unmasking. Among gliomas of the astrocytic lineage, there was an inverse relationship between the percentage of $\mathrm{PTEN}^{+}$tumor cells and malignancy grade. Low-grade astrocytomas (4 pilocytic astrocytomas and 2 grade II astrocytomas) showed immunoreactivity in almost every tumor cell, especially in the dense glial fibers in the pilocytic astrocytomas (Fig. 2A). PTEN immunoreactivity was completely blocked by preincubation of the antibody with purified antigen. The anaplastic astrocytomas showed variable PTEN expression $(0 \%-65 \%$ positive cells) (Fig. $2 \mathrm{~B})$. No PTEN immunoreactivity was detectable in 14 of 21 GBMs (67\%) (Fig. 2C).

Among tumors of oligodendroglial lineage, a similar pattern of PTEN expression was observed, with $37 \%$ to $100 \%$ positive cells $($ median $=56 \%$ ) in low-grade oligodendrogliomas (Fig. 2D) and $0 \%$ to $19 \%$ (median $=9 \%$ ) in malignant oligodendrogliomas (Fig. 2E). In five cases of malignant, mixed oligoastrocytomas, cytoplasmic staining was present in tumor cells with morphologic features of immature oligodendroglia (small round nuclei; scant cytoplasm) as well as in those with astrocytic features (elliptical nuclei; short cytoplasmic processes) (Fig. 2F).

Fig. 3 shows a dot plot of the percentage of PTEN ${ }^{+}$ cells in the six subclasses of gliomas. We compared the distributions of these percentages using an exact version of the Wilcoxon rank-sum test. According to the hypoth- esis that an inverse relationship exists between tumor grade and PTEN expression, we performed one-sided tests to compare low-grade astrocytoma versus anaplastic astrocytoma $(P=0.0006)$, anaplastic astrocytoma versus GBM $(P=0.0042)$, and low-grade oligodendroglioma versus malignant oligodendroglioma $(P=$ 0.0048). All these differences are significant at the 0.05 level. The less meaningful comparison of low-grade astrocytoma versus malignant mixed oligoastrocytoma $(P=0.0022)$ showed a significant difference, but the low-grade oligodendroglioma versus malignant mixed oligoastrocytoma comparison $(P=0.056)$ did not quite reach significance. The proportions of $\mathrm{PTEN}^{+}$cells among the six glioma subclasses were compared using analysis of covariance (ANCOVA) on the logit-transformed proportions. The effects of the covariates - age, gender, and treatment history- were not significant for any of these comparisons.

We observed the same pattern and specificity of staining with antisera raised against the $\mathrm{N}$-terminal portion of the PTEN molecule and against the C-terminal portion. The results shown in Table 1 were obtained using hightiter, C-terminal antibody UT139. Taken together, these comparisons show an inverse relationship between PTEN immunoreactivity and malignancy grade in both astrocytomas and oligodendrogliomas, consistent with a role for PTEN as a tumor suppressor gene, the expression of which declines during glioma progression.

\section{PTEN Immunocytochemistry in Nonneoplastic Brain Tissue}

To examine the distribution of PTEN expression in nonneoplastic brain tissue, we performed immunoperoxidase staining of brain sections from the following sources: hippocampus and temporal neocortex removed from an adult patient during surgery for epilepsy, putamen obtained at autopsy from a patient with Alzheimer's Disease, frontal lobe autopsy specimen from a patient with multiple sclerosis, and three human fetal brain specimens (frontal lobe) at 16, 23, and 27 weeks' gestation.

PTEN immunoreactivity was not detectable in either neurons or glia in the adult brain sections. (Fig. 2G shows results in the dentate gyrus of hippocampus.) In fetal brain, however, PTEN immunoreactivity was present. In the 27-week specimen, PTEN immunoreactivity was present throughout fetal layer II, the most superficial part of the cortical plate (Mrzijak et al., 1988). In addition, PTEN immunoreactivity was visible in the cell bodies and apical dendrites of pyramidal neurons in fetal layer V. Only subpopulations of these neurons were positive (Fig. 2H). At 16 and 23 weeks' gestation, PTEN immunoreactivity was present throughout the cortical plate region (Fig. 2I). Faint brown staining was visible in the marginal, subplate, and intermediate zones, indicating that PTEN expression was absent there, or very low. No PTEN expression was seen in the primitive neuroepithelial cells of the ventricular germinal matrix present in the 16-week fetal brain specimen. These results indicate that PTEN is developmentally regulated in the human cerebrum. 

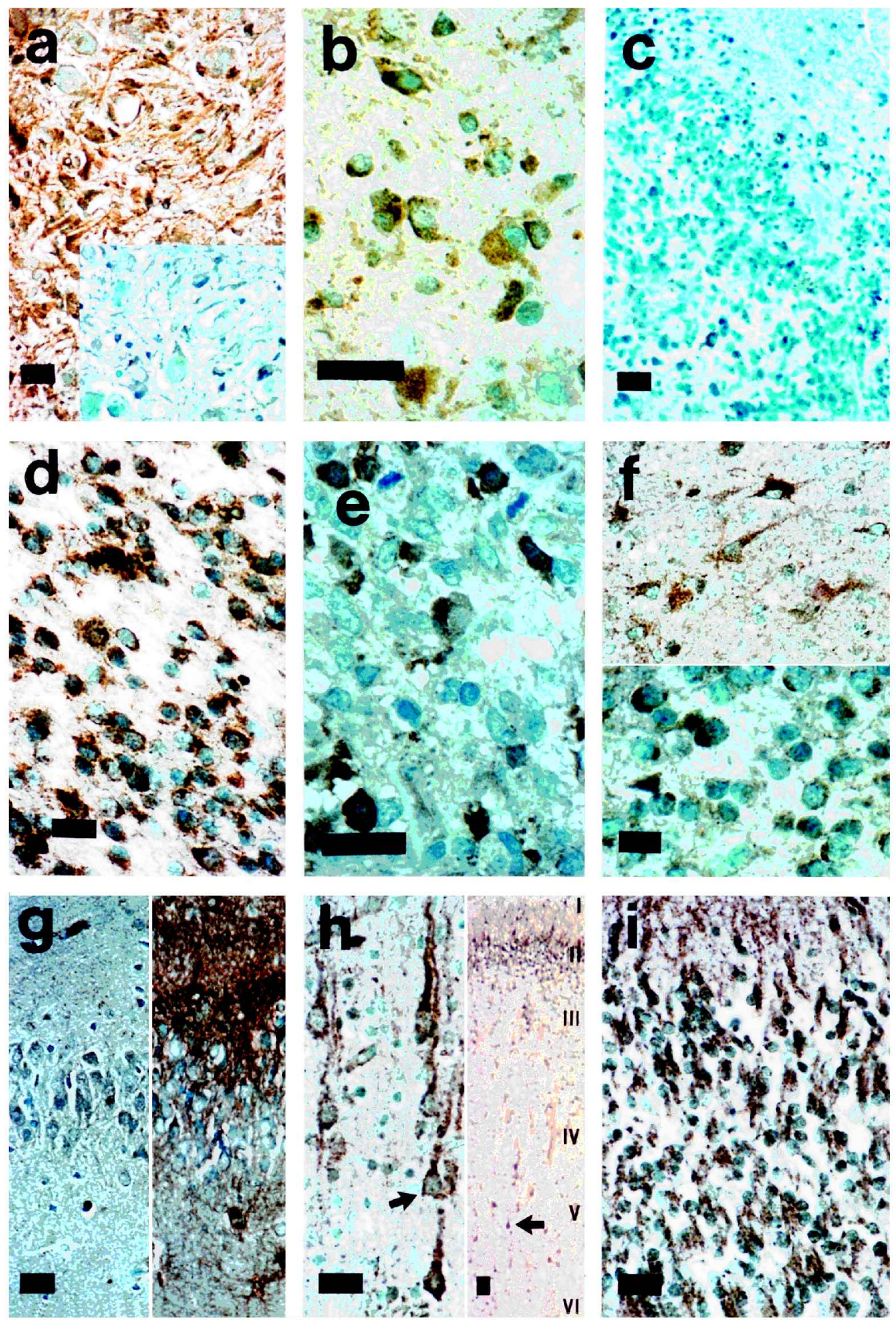

Fig. 2. Immunoperoxidase staining of PTEN in human gliomas (A-F) and nonneoplastic brain tissue (G-I). A. Pilocytic astrocytoma (case L28). Inset shows competitive blocking of PTEN immunoreactivity by preadsorption of antibody with PTEN-GST fusion peptide. Elliptical structures are granular bodies. B. Anaplastic astrocytoma (case A34). C. Glioblastoma multiforme (case U42). A focus of tissue necrosis is visible in the upper right corner. D. Low-grade oligodendroglioma (case O18). E. Malignant oligodendroglioma (case O11). Two mitotic cells are visible in the upper part of the micrograph. F. Malignant mixed oligoastrocytoma (case A26) with PTEN immunoreactivity in both astrocytic component (upper panel) and oligodendroglial component (lower panel). G. Absence of PTEN immunoreactivity in dentate gyrus of adult hippocampus (left panel). Adjacent section stained with antiglial fibrillary acidic protein antibody (right panel). H. Low power view of 27-week cerebral cortex showing PTEN immunoreactivity in fetal layer II (external granular layer) and in subpopulations of pyramidal neurons (arrows) of layer IV (right panel). Roman numerals indicate cortical layers (Mrzijak et al., 1988). High power view of PTEN ${ }^{+}$pyramidal neurons in layer IV (left panel). I. PTEN immunoreactivity in the cortical plate of 23-week fetal brain. A portion of the marginal layer is visible at the upper edge of the micrograph. Scale bars, $10 \mu \mathrm{m}$. 


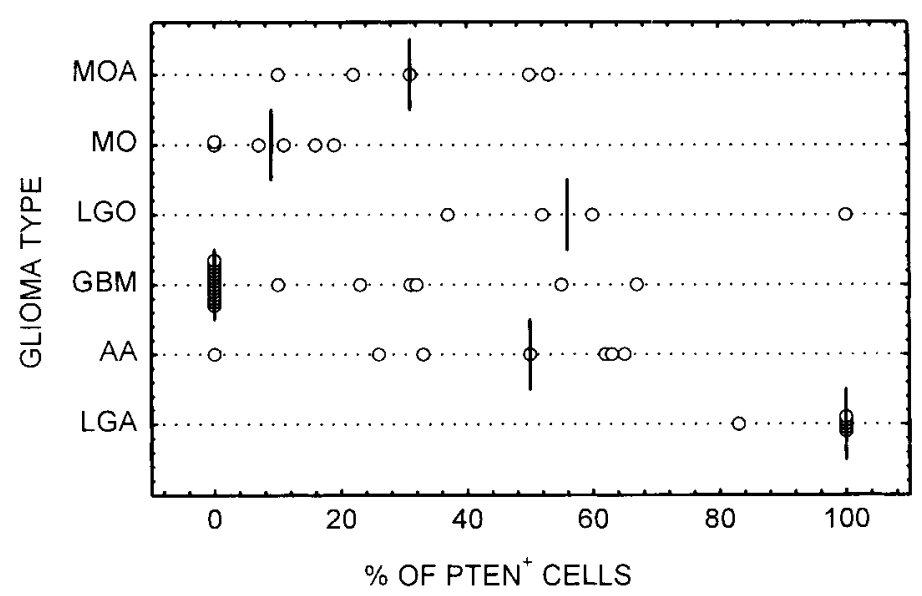

Fig. 3. PTEN protein expression in 49 gliomas. Dot plot shows the distribution of percentages of PTEN ${ }^{+}$tumor cells in six glioma subclasses: LGA, low-grade astrocytoma (pilocytic and grade 2 astrocytomas); AA, anaplastic astrocytoma; GBM, glioblastoma multiforme; LGO, low-grade oligodendroglioma; $M O$, malignant oligodendroglioma; MOA, malignant mixed oligoastrocytoma. The median values for each glioma type are indicated by vertical bars.

\section{Correlation of PTEN Genotype and Expression in GBM}

In 21 GBM cases, the genotype of the PTEN coding region and $\mathrm{LOH}$ status were known (Table 1). Six cases (U49, U48, U72, U42, U71, U23) had LOH on 10q and a chain-terminating mutation in the PTEN gene (three stop codons, one frameshift, and two splice donor site variants). No PTEN immunoreactivity was found in these cases, the expected result for null mutations. Four cases (U52, U80, U50, U15) had LOH on 10q and a PTEN missense mutation. Three of these missense mutants showed no protein expression in the tumor cells. One GBM (U15) with a missense mutation (an M $>$ R amino acid substitution at codon 35 in exon 2) did show PTEN protein expression in $31 \%$ of tumor cells. Among the 11 GBMs with wild-type PTEN, tumor cell protein was absent in 5 cases and present in 6 cases. We compared the distributions of the percentages of $\mathrm{PTEN}^{+}$tumor cells in GBMs with wild-type and mutant PTEN using the Wilcoxon rank-sum test. PTEN-mutant tumors showed significantly diminished PTEN protein expression compared with wild-type cases $(P=0.00423)$.

\section{Discussion}

In gliomas of astrocytic lineage, the inverse relationship we observed between PTEN protein expression and histopathology grade is consistent with a role for PTEN as a tumor suppressor gene that becomes inactivated during progression to end-stage GBM. The variation in PTEN expression among anaplastic astrocytomas suggests that PTEN loss may be a marker for tumors that have already made this transition. Furthermore, the presence of peripherally located subpopulations of PTEN $^{+}$tumor cells in some GBM cases supports the idea that loss of PTEN expression confers a growth advantage to the mutant cells and permits their clonal expansion.
In a previously reported sequence analysis, PTEN gene mutations were not found in oligodendrogliomas, either benign or malignant (Rasheed et al., 1997). In the present study, we found PTEN protein expression to be diminished in malignant oligodendrogliomas compared with low-grade oligodendrogliomas, a pattern similar to that found in astrocytomas. Taken together, these findings suggest that down regulation of PTEN is a factor in malignant progression of oligodendrogliomas, even without PTEN gene mutation.

Our observation that PTEN is expressed in fetal cerebral cortex, coupled with the known inhibitory effect of overexpressed PTEN on cell migration in wound healing assays (Tamura et al., 1998), suggests that PTEN may play a role in migration of differentiating neurons and/or glia from the ventricular germinal matrix to the neocortex during embryogenesis. In support of this concept, germline mutations in PTEN occur in patients with Lhermitte-Duclos disease, a condition wherein hamartomatous masses of hypertrophic ganglion cells replace the normal layers of granule and Purkinje neurons in the cerebellum (Liaw et al., 1997). The absence of PTEN expression in the proliferative neuroepithelium of the 16week brain suggests that PTEN may be more important in regulating cell migration than in regulating proliferation, at least during neural development. Somatically acquired PTEN mutations encoding inactive versions of PTEN would likely contribute to the well-known hypermotility of tumor cells in human gliomas.

The prognosis for patients with gliomas correlates tightly with histopathologic tumor grade (reviewed in Kleihues and Cavenee, 1997). A study of PTEN expression, as measured by reverse transcriptase-polymerase chain reaction, in 89 gliomas showed that survival times were significantly longer in patients whose tumors expressed high levels of PTEN mRNA (Sano et al., 1999). Although our study lacked sufficient statistical power to establish PTEN as an independent predictor of clinical outcome, the close correlation we found between PTEN immunoreactivity and tumor grade indicates that 
loss of PTEN expression is a marker for increased aggressiveness in both oligodendrogliomas and astrocytomas.

Correlation of PTEN immunoreactivity with PTEN genotype in 21 GBMs revealed that cell protein levels were undetectable or markedly diminished, not only in PTEN-mutant tumors, but also in most wild-type PTEN tumors. One genetic mechanism that may account for decreased or absent PTEN expression in tumors with wild-type PTEN sequence is haploinsufficiency. Because all but one of our wild-type PTEN GBMs had LOH on $10 \mathrm{q}$, gene transcription from the single remaining allele may have dropped cell protein levels below the level of immunodetection. According to a theoretical model of stochastic gene expression, inactivation of a single allele in diploid cells decreases the probability of gene expression from the remaining allele (Cook et al., 1998).

Alternatively, these $\mathrm{PTEN}^{-}$tumors may have arisen from glial cells that originally expressed only one of two existing PTEN alleles, the other having been functionally silenced during the course of embryonic development (monoallelic expression). Monoallelic expression of the insulin-like growth factor type 2 (IGF2) gene was demonstrated in a study of Wilms' tumors using allelespecific, in situ hybridization probes (Ohlsson et al., 1998). Within individual tumors, some cells preferentially expressed one insulin-like growth factor type 2 allele, while other cells expressed the other allele, exclusively. If a population of glioma cells expressed PTEN in a monoallelic fashion and the expressed allele became deleted (chromosome instability may have arisen from previously acquired mutations in other genes), then PTEN protein would disappear altogether. This series of events might confer a growth advantage, leading to the clonal expansion of glioma cells devoid of PTEN protein yet still retaining the wild-type PTEN gene sequence.

Another mechanism of ablating PTEN protein expression in cells without a coding sequence mutation is by methylation of the promoter. In support of this concept, one group of investigators restored PTEN mRNA expression in a prostate carcinoma xenograft by treatment with 5-azadeoxycytidine, a demethylating agent (Whang et al., 1998). PTEN expression may also be down regulated by transacting factors like transforming growth factor (TGF)- $\beta$, which is produced by glioma cells and which decreases PTEN gene expression (Li and Sun, 1997).

We cannot exclude the possibility that the PTEN missense mutants were expressed but remained unrecognized by our antibodies, which were raised against wild-type proteins. Nevertheless, our immunocytochemistry results indicate that suppressed expression of PTEN in GBM is more prevalent than predicted from mutation frequencies. A broader implication of this work is that a low mutation frequency in primary tumors does not exclude a candidate gene as a physiologically relevant tumor suppressor.

\section{Acknowledgments}

The authors thank Patricia Maness (University of North Carolina) and Scott Rogers (University of Utah) for helpful advice on immunology, David James (Mayo Clinic) for providing cDNAs, Jeanette Townsend, Tom Parks (University of Utah), and Eric Holland (University of Texas) for critical discussions, and the Biostatistics Resource Core Facility (Huntsman Cancer Institute) for statistical analysis.

\section{References}

Cook, D.L., Gerber, A.N., and Tapscott, S.J. (1998) Modeling stochastic gene expression: Implications for haploinsufficiency. Proc. Natl. Acad. Sci. U.S.A. 95, 15641-15646.

Fults, D., and Pedone, C. (1993) Deletion mapping of the long arm of chromosome 10 in glioblastoma multiforme. Genes Chromosomes Cancer 7 , 173-177.

Fults, D., Pedone, C.A., Thompson, G.E., Uchiyama, C.M., Gumpper, K.L., Iliev, D., Vinson, V.L., Tavtigian, S.V., and Perry, W.L. (1998) Microsatellite deletion mapping on chromosome $10 \mathrm{q}$ and mutation analysis of MMAC1, FAS, and MXI1 in human glioblastoma multiforme. Int. J. Oncol. 12, 905-910.

Furnari, F.B., Huang, H.S., and Cavenee, W.K. (1998) The phosphoinositol phosphatase activity of PTEN mediates a serum-sensitive 11 growth arrest in glioma cells. Cancer Res. 58, 5002-5008.

Haas-Kogan, D., Shalev, N., Wong, M., Mills, G., Yount, G., and Stokoe, D. (1998) Protein kinase B (PKB/Akt) activity is elevated in glioblastoma cells due to mutation of the tumor suppressor PTEN/MMAC. Curr. Biol. 21, 1195-1198.

Hsu, S.M., Raine, L., and Fanger, H. (1981) Use of avidin-biotin peroxidase complex $(A B C)$ in immunoperoxidase techniques: A comparison between $A B C$ and unrelated antibody (PAP) procedures. J. Histochem. Cytochem. 29, 577-580.

Kleihues, P., Burger, P.C., and Scheithauer, B.W. (1993) Histological Typing of Tumours of the Central Nervous System. Berlin: Springer-Verlag.
Kleihues, P., and Cavenee, W.K. (Eds.) (1997) Pathology and Genetics of Tumours of the Nervous System. Lyon, France: International Agency for Research on Cancer.

Li, D.M., and Sun, H. (1997) TEP1, encoded by a candidate tumor suppressor locus, is a novel protein tyrosine phosphatase regulated by transforming growth factor $\beta$. Cancer Res. 57, 2124-2129.

Li, J., Yen, C., Liaw, D., Podsypanina, K., Bose, S., Wang, S.I., Puc, J., Miliaresis, C., Rodgers, L., McCombie, R., Bigner, S.H., Giovanella, B.C., Ittmann, M., Tycko, B., Hibshoosh, H., Wigler, M.H., and Parsons, R. (1997) PTEN, a putative protein tyrosine phosphatase gene mutated in human brain, breast, and prostate cancer. Science 275, 1943-1947.

Liaw, D., Marsh, D.J., Li, J., Dahia, P.L.M., Wang, S.I., Zheng, Z., Bose, S., Call, K.M., Tsou, H.C., Peacocke, M., Eng, C., and Parsons, R. (1997) Germline mutations of the PTEN gene in Cowden disease, an inherited breast and thyroid cancer syndrome. Nat. Genet. 16, 64-67.

Liu, W., James, C.D., Frederick, L., Aldrete, B.E., and Jenkins, R.B. (1997) PTEN/MMAC1 mutations and EGFR amplification in glioblastomas. Cancer Res. 57, 5254-5257.

Maehama, T., and Dixon, J.I. (1998) The tumor suppressor, PTEN/MMAC1, dephosphorylates the lipid second messenger, phosphatidylinositol 3,4,5trisphosphate. J. Biol. Chem. 273, 13375-13378.

Mrzijak, L., Uylings, H.B.M., Kostovic, I., and Van Eden, C.G. (1988) Prenatal development of neurons in the human prefrontal cortex: A qualitative Golgi study. J. Comp. Neurol. 271, 355-386. 
Myers, M.P., Stolarov, J.P., Eng, C., Li, J., Wang, S.I., Wigler, M.H., Parsons, R., and Tonks, N.K. (1997) P-TEN, the tumor suppressor from human chromosome 10q23, is a dual-specificity phosphatase. Proc. Natl. Acad. Sci. U.S.A. 94, 9052-9057.

Noble, M. (1996) Steps toward a cellular biological analysis of human gliomas. In: Raffel, C., and Harsh. G.R. (Eds.), The Molecular Basis of Neurosurgical Disease. Baltimore: Williams and Wilkins. Vol. 8, pp. 87-97.

Ohlsson, R., Tycko, B., and Sapienza, C. (1998) Monoallelic expression: “There can only be one." Trends Genet. 14, 435-438.

Rasheed, B.K.A., Stenzel, T.T., McLendon, R.E., Parsons, R., Friedman, A.H., Friedman, H.S., Bigner, D.D., and Bigner, S.H. (1997) PTEN gene mutations are seen in high-grade but not in low-grade gliomas. Cancer Res. 57, 4187-4190.

Sano, T., Lin, H., Chen, X., Langford, L.A., Koul, D., Bondy, M.L., Hess, K.R., Myers, J.N., Hong, Y.K., Yung, W.K.A., and Steck, P.A. (1999) Differential expression of MMACIPTEN in glioblastoma multiforme: Relationship to localization and prognosis. Cancer Res. 59, 1820-1824.

Steck, P.A., Pershouse, M.A., Jasser, S.A., Yung, W.K.A., Lin, H., Ligon, A.H., Langford, L.A., Baumgard, M.L., Hattier, T., Davis, T., Frye, C.,
Hu, R., Swedlund, B., Teng, D.H.F., and Tavtigian, S.V. (1997) Identification of a candidate tumour suppressor gene, MMAC1, at chromosome $10 q 23.3$ that is mutated in multiple advanced cancers. Nat. Genet. 15, 356-362.

Suzuki, A., de la Pompa, J.L., Stambolic, V., Elia, A.J., Sasaki, T., Barrantes, I.B., Ho, A., Wakeham, A., Itie, A., Khoo, W., Fukumoto, M., and Mak, T.W. (1998) High cancer susceptibility and embryonic lethality associated with mutation of the PTEN tumor suppressor gene in mice. Curr. Biol. 21, 1169-1178.

Tamura, M., Gu, J., Matsumoto, K., Aota, S., Parsons, R., and Yamada, K.M. (1998) Inhibition of cell migration, spreading, and focal adhesions by tumor suppressor PTEN. Science 280, 1614-1617.

Wang, S.I., Puc, J., Li, J., Bruce, J.N., Cairns, P., Sidransky, D., and Parsons, R. (1997) Somatic mutations of PTEN in glioblastoma multiforme. Cancer Res. 57, 4183-4186.

Whang, Y.E., Wu, X., Suzuki, H., Reiter, R.E., Tran, C., Vessella, R.L., Said, J.W., Isaacs, W.B., and Sawyers, C.L. (1998) Inactivation of the tumor suppressor PTEN/MMAC1 in advanced human prostate cancer through loss of expression. Proc. Natl. Acad. Sci. U.S. A. 95, 5246-5250. 